\title{
Sistemas semióticos, artefatos cognitivos, Umwelt - uma contribuição ao Design da Informação
}

\author{
Semiotic systems, cognitive artefacts, Umwelt - a contribution to Information Design \\ João Queiroz
}

signo, sistemas semióticos, artefatos semióticos, Umwelt

\begin{abstract}
O desenvolvimento e a análise de "ambientes, nichos e paisagens semióticas" (semiotic landscapes) pode caracterizar com precisão parte das atividades do Design da Informação. Este trabalho explora introdutoriamente esta perspectiva, através das noções de "artefato cognitivo e semiótico", "sistema semiótico" e Umwelt. Ele se inscreve em um domínio teórico em que associa processos semióticos, tecnologias e artefatos cognitivos, e baseia-se em tratamentos empíricos, em Ecologia Comportamental, Etologia, e Semiótica Cognitiva.
\end{abstract}

sign, semiotic systems, semiotic artifacts, Umwelt

The development and analysis of 'environments, niches and semiotic landscapes' may accurately characterize the activities of Information Design. This introductory paper explores this perspective through the notions of 'cognitive and semiotic artifacts', 'semiotic system' and Umwelt. It is presented from within a theoretical field, relating semiotic processes, cognitive technologies and artifacts, and it is based on empirical treatment in Behavioral Ecology, Ethology, and Cognitive Semiotics.

\section{Introdução}

A ocupação com a criação, desenvolvimento, e "otimização" de paisagens semióticas (semiotic landscapes), de interfaces para dispositivos de navegação a nichos de imersão ubíqua (Grau 2003), e a investigação dos processos interpretativos que têm lugar em tais ambientes, pode caracterizar com precisão importantes domínios de competência do Design da Informação. Interessa-me abordar introdutoriamente algumas noções capazes de fornecer um desenvolvimento consistente a esta perspectiva. Signo, sistema semiótico, artefato cognitivo e Umwelt estão entre as principais noções introduzidas aqui. Estes conceitos atraem pesquisadores de diversas áreas —Ciências Cognitivas, Inteligência Artificial (IA), Sistemas Inteligentes (SI), Ecologia Comportamental, Design de Interfaces- e devem prover uma "bateria de problemas e noções" para o desenvolvimento desta perspectiva.

A relação entre Signo e Umwelt, "artefato", "nicho cognitivo", e "tecnologia cognitiva", tem sido destacada, especialmente nas discussões sobre "artefatos simbólicos", por cientistas e filósofos (e.g. Kirsh, 2010, 2009; Sinha, 2009; Sterelny, 2008; Zhang \& Wang, 2009; Clark, 2008, 2006, 2003; Emmeche, 2006; Dascal, 2002; Hutchins, 1999; Norman, 1993), e tem importantes precursores (Peirce, Dewey, Wittgenstein, Vigotski, George Mead). Meu argumento é que pode tratar-se de uma estratégia heuristicamente poderosa a introdução destas pesquisas em Design da Informação. Este é obviamente um trabalho especulativo, mais interessado nas possibilidades criadas pela aproximação entre diferentes domínios de investigação, e caracterização da "área" em termos de novos problemas e metáforas epistêmicas. Um desenvolvimento ulterior das perspectivas introdutoriamente exploradas aqui deve incluir descrição e análise de casos e exemplos. O trabalho está dividido nas seguintes seções: (i) noção Peirceana de semiose e tipos fundamentais de signos; (ii) sistemas semióticos; (iii) artefato cognitivo e semiótico; (iv) Umwelt; (v) algumas implicações. 


\section{Lógica do signo de C.S.Peirce e tipos fundamentais de signos}

Para C.S.Peirce ${ }^{1}$, semiose (meaning process) é a "ação do signo" (Short, 2007; Queiroz \& Merrell, 2006, 2009). O fenômeno é descrito como uma relação irredutivelmente triádica (relação indecomponível) que conecta um Signo a seu Objeto para um Interpretante (efeito em um intérprete). Trata-se de uma relação em que um intérprete sofre o efeito de um Objeto por meio de um Signo. Em diversos trabalhos, o Signo é pragmaticamente definido por Peirce como um meio para a comunicação de um hábito, uma "regra de ação", ou uma "regularidade", incorporados (embodied) no Objeto que o Signo representa, de modo a restringir o comportamento do intérprete (ver Queiroz \& El-Hani, 2006a,b; De Tienne, 2003; Bergman, 2000).

[...] um Signo pode ser definido como um Meio para a comunicação de uma Forma. Como um meio, o Signo está essencialmente em uma relação triádica, para o seu Objeto que o determina, e para o seu Interpretante que ele determina. [...] Aquilo que é comunicado do Objeto através do Signo para o Interpretante é uma Forma; o que significa dizer, não é nada como um existente, mas é um poder, é o fato de que alguma coisa aconteceria sob certas condições (EP 2.544, n.22).

O que é comunicado a partir do Objeto, por meio do Signo, para um intérprete, é o fato de que alguma coisa deveria acontecer sob certas condições. São três os tipos fundamentais de Signos: icônicos, indexicais e simbólicos. Eles, aproximadamente, correspondem a relações de similaridade, de contigüidade física, e de lei que podem ser estabelecidas entre um Signo e seu Objeto.

O ícone é definido como um Signo (S) que se baseia em relações de similaridade (CP 2.276), e que pode prescindir de qualquer correlação espaço-temporal com um objeto $(\mathrm{O})$ existente (CP 5.73). O ícone é portanto dependente de suas próprias propriedades. São as qualidades que pertencem ao Signo que determinam sua interpretação de tal forma que qualquer objeto que tem qualidades similares pode ser interpretado como seu Objeto. O signo icônico "serve como um signo simplesmente por exibir a qualidade de que se serve para significar" (NEM 4: 242). Ele comunica um hábito incorporado em O para I (interpretante) de modo a restringir o comportamento do intérprete como o resultado de certas qualidades que compartilha com $\mathrm{O}$. Em contraste, se $\mathrm{S}$ é um Signo de $\mathrm{O}$ em razão de uma relação diádica com $\mathrm{O}$, então ele é índice de $\mathrm{O}$. Nesse caso, $\mathrm{S}$ e O estão relacionados através de uma "conexão física direta" (CP 1.372). A noção de co-variação espaço-temporal é uma propriedade característica dos processos indexicais. Um signo indexical comunica para I um hábito incorporado em $\mathrm{O}$ como um resultado de uma conexão física direta com O. Finalmente, o símbolo é um Signo que está relacionado ao seu Objeto em virtude de uma lei; ele comunica uma lei a seu intérprete. Símbolos são capazes de representar "coisas" que não precisam existir de fato, ou que existem mas não estão perceptualmente manifestas, que jamais existiram, ou, ainda, em termos mais antroposemióticos, que não podem sequer ser intuitivamente concebidas (e.g. "estranhos" objetos das lógicas não-clássicas, objetos matemáticos, criaturas imaginárias, etc.). Uma importante propriedade dos símbolos é que restrições impostas à presença espaço-temporal do Objeto representado pelo índice, que ele conecta coincidentemente, não tem lugar em processos simbólicos. Segundo Peirce, em um símbolo, a relação entre $S$ e $O$ é logicamente dependente do terceiro termo da relação, do Interpretante: "o símbolo está conectado com o objeto em virtude da idéia de uma mente que usa o símbolo, sem a qual tal conexão não existiria" (CP 2.299). A distinção "não-simbólico/simbólico" é importante porque restrições impostas a processos indexicais, à presença espaço-temporal do Objeto representado pelo índice, que ele conecta coincidentemente, não tem lugar em processos simbólicos.

\section{Sistemas semióticos e classes de signos}

Um sistema semiótico é um sistema que produz, transmite e interpreta signos de diferentes tipos. De acordo com Fetzer (1997: 358; 1988), um sistema semiótico é "causalmente afetado pela presença de um signo porque está para algo iconicamente, indexicalmente, simbolicamente". Eles exibem comportamento auto-corretivo, ou algum tipo de atividade orientada por um propósito (Ransdell, 1977: 162). Para descrever mais detalhadamente sistemas semióticos, deve-se especificar uma morfologia de sistemas definida pelas modalidades examinadas acima — sistemas semióticos icônicos, indexicais e simbólicos (Queiroz \& El-Hani, 2006b). Em termos sumários,

${ }^{1}$ A obra de Peirce será citada como CP (seguido pelo número do volume e parágrafo), The Collected Papers of Charles S. Peirce, Peirce 1931-1935; EP (seguido pelo número do volume e página), The Essential Peirce, Peirce 1893-1913; e NEM (seguido pelo número do volume e página), New elements of mathematics by Charles S. Peirce, Peirce 1976. 
sistemas semióticos usam signos como meios para comunicação de "padrões de similaridade", "correlações espaço-temporais" e "relações legaliformes".

Um sistema icônico tem seu comportamento regulado por uma certa qualidade que ele interpreta como compartilhada entre o Signo e seu Objeto. Em termos cognitivos, ele atua no reconhecimento sensório de estímulos de qualquer modalidade, e em associações de analogia. Um sistema indexical, de outro modo, é sensível a covariações espaço-temporais. Ele é causalmente afetado por uma correlação incorporada no Objeto como uma coincidência comunicada pelo Signo. Se um signo indexical comunica um hábito incorporado no Objeto, como resultado de uma conexão física direta entre Signo e Objeto, então um sistema indexical é causalmente afetado pelos eventos e ocorrências interpretados como semioticamente coincidentes. Para um sistema simbólico, a forma comunicada do Objeto através do Signo é uma "relação legal" (legaliforme) entre um certo tipo de Signo e um certo tipo de Objeto. O símbolo comunica um hábito incorporado no Objeto como resultado de uma lei que relaciona Signo e Objeto - "um símbolo é uma lei, ou regularidade do futuro indefinido" (CP 2.293). Diferente das restrições impostas a processos indexicais, à presença espaço-temporal do Objeto representado pelo índice, os processos simbólicos não dependem logicamente do Objeto.

Duas questões podem ser feitas neste ponto: que relação pode-se estabelecer entre signos, sistemas semióticos e artefatos cognitivos?

\section{Artefatos cognitivos e semióticos}

Os artefatos cognitivos modificam habilidades cognitivas, podendo alterá-las radicalmente, e podem ser divididos em diversas categorias: "material \& mental" (Norman, 1993), "planejado para" \& "oportunístico" (Hutchins, 1999), "transparente \& opaco" (Clark, 2003). Os exemplos incluem modelos, notações, sinais, marcadores, mensuradores e, mais trivialmente, calendários, listas de compras, placas de trânsito, bandeiras. As tecnologias empregadas envolvem lapis e papel, ábacos, calculadoras, computadores, internet, celulares, GPSs, e outras novidades tecnológicas. Segundo Clark (2003), o resultado do uso de artefatos cognitivos é notável: o espaço, acoplado, torna-se cada vez mais inteligente, criando seres híbridos (simbiontes de tecnologias), e a linguagem é o mais poderoso dos artefatos. Para Hutchins, a sugestão de Clark deve estar correta. Segundo Hutchins (1999), a linguagem é o mais importante artefato cognitivo, e um sistema "absolutamente fundamental para consciência e para o que significa ser humano". Para Tomasello (Tomasello et al., 2005), interessado nas consequências de um tipo particular de artefato, os "símbolos" funcionam de modo a permitir (ou facilitar o aparecimento de) certos tipos de interações sociais, alterando radicalmente as competências cognitivas de seus usuários. (Tomasello está interessado nos requisitos sociogênicos para explicar o aparecimento de algumas propriedades necessárias [e.g. "theory of mind", "joint attention"], em primatas não-humanos e em infantes, e nas diversas escalas temporais [filogenética, histórica, ontogenética] requeridas para explicação do aparecimento de símbolos.)

O que mais me interessa aqui são as consequências que a aproximação entre as noções de Signo e artefato cognitivo podem produzir, sugerindo o que pode ser chamado de "artefato semiótico" e cujos componentes podem ser classificados em artefatos icônicos, indexicais, simbólicos.

Os artefatos semióticos permitem a realização de diversas tarefas semióticas. A idéia de que certas modalidades semióticas são especialmente adequadas a tarefas específicas deve parecer trivial. É dificil conceber uma operação aritmética (e.g. exponenciação) realizada por meio da manipulação de um conjunto de signos usado para designar eventos espaço-temporalmente coincidentes. Ou, ao contrário, tentar representar eventos temporalmente distantes através de entidades que variam espaço-temporalmente com seus objetos (e.g. fumaça > fogo). Parece natural escolher um sistema de linhas e pontos para localizar a estação de metrô mais próxima, ou escolher uma notação com regras de combinatoriedade e recursividade para dividir e exponenciar com números. Certos Signos (ou sistemas de Signos) são apropriados para certas tarefas, e há tarefas que não podem ser concebidas se dissociadas de certos tipos de Signos. Signos baseados em leis de recursão permitem explosões combinatoriais. Signos que causalmente decorrem de eventos locais permitem inferir a presença imediata de seus Objetos com alta confiabilidade. Signos que permitem visualizar relações espaciais são excelentes candidatos à representação de distâncias e proporções. 
Tais modalidades de artefatos (semióticos) constroem espaços, ou nichos, especializados. A noção de que estamos imersos em nichos ou paisagens mais ou menos estruturadas de signos que funcionam como artefatos é uma idéia ainda pouco explorada. Suas conseqüências mais radicais indicam que atividades cognitivas diversas dependem da manipulação de tipos específicos de signos, e que estes proveem "atalhos" para solução de problemas específicos. Também indicam que sistemas semióticos estão imersos em ambientes de short-cuts construídos e/ou selecionados para agir e obter informação sobre o ambiente. Por exemplo, os diagramas são a principal, senão a única, forma de adquirirmos nova informação sobre relações espaciais (ver Stjernfelt, 2007). Diagramas e grafos, que representam seus objetos através das relações entre as partes que os constituem, são artefatos semióticos projetados para revelar informação sobre relações. Parece um truismo afirmar que a maneira mais eficaz de representar relações espaciais é por meio de diagramas, como mapas ou frames. Embora uma descrição de relações espaciais possa basear-se em estruturas linguísticas, por exemplo, sabemos que a melhor tecnologia baseia-se na manipulação de linhas, arcos, e vértices.

Mas uma explicação robusta sobre as restrições que atuam na construção de nichos semióticos deveria atuar em diversos níveis de descrição. Vou introduzir rapidamente a noção de Umwelt, capaz de explicar as restrições que atuam no nível de operações do equipamento sensório-efetor.

\section{Umwelt, nicho cognitivo e paisagem semiótica}

Em 1909, Jakob von Uexküll desenvolveu o conceito de Umwelt como o "mundo fenomenal" sensório-efetor que cada espécie animal possui, o ambiente percebido através de suas interações. O Umwelt de uma criatura define o espectro de posições que ela pode ocupar na esfera biológica. Essa abordagem introduziu um conjunto de ferramentas conceituais, fornecendo importantes bases à biossemiótica, popularizada mais tarde por Thomas Sebeok (ver Queiroz et al., prelo). Umwelt pode ser definido como o mundo semiótico dos organismos, ou o aspecto fenomenal do ambiente selecionado pelo equipamento sensório-motor da espécie, de acordo com suas necessidades biológicas (J. von Uexküll, 1940; T. von Uexküll, 1982). Segundo Emmeche (2006), deve-se distinguir: (1) o habitat objetivo do organismo (externo), que pode ser descrito por um observador; (2) o nicho do organismo, como a função ecológica das espécies em um ecossistema, (3) o Umwelt - mundo experienciado pelo organismo.

Em ecologia, o conceito de nicho ecológico é descrito como a posição que uma espécie, ou uma população, ocupa no ecossistema. Esse conceito inclui, além do espaço físico em que atua o organismo, seu papel funcional na comunidade e sua posição nos gradientes ambientais (temperatura, $\mathrm{pH}$, solo, etc). $\mathrm{O}$ nicho ecológico portanto depende não apenas de seu sítio, mas do que ele faz, e a forma como é constrangido por outras espécies e por fatores abióticos. Um princípio importante da Ecologia é que cada espécie possui um nicho distinto. Não há espécies idênticas, e cada espécie tem atributos distintos de forma e função que determinam as condições que podem tolerar.

Estas idéias têm importantes implicações, se estiver correta a suposição de que estamos imersos em processos e estruturas semióticas. Segundo Clark (2006) os ambientes, que podemos chamar de "paisagens semióticas", estão estruturados como "nichos cognitivos", feitos de materiais e estruturas construídas para transformar os problemas no espaço de maneira a auxiliar a ação, em geral.

\section{Implicações}

De acordo com a perspectiva preliminar introduzida aqui, o Design da Informação se interessa pela concepção e construção de nichos semioticamente especializados. Esta orientação corresponde à introdução de uma nova bateria de metáforas epistemológicas, e a uma nova lista de problemas, modelos e teorias. Devem estar entre as perguntas mais relevantes e frequentes: como artefatos semióticos melhoram as performances de seus usuários? Como classificá-los? Entre as competências inauguradas pela criação e uso de novos artefatos, qual a importância de diferentes modalidades de artefatos (icônico, indexical, simbólico)? Em outras palavras, como sistemas icônicos, indexicais, ou simbólicos, atuam, provendo competências cognitivas muito distintas? 
Para classificar a variedade de eventos que causalmente atuam sobre sistemas semióticos, Peirce propôs uma morfologia bastante conhecida -ícones, índices, símbolos. Não explorei aqui muitas propriedades que caracterizam esta divisão, em termos fenomenológicos (teoria das categorias), lógicos ou inferenciais (abdução, dedução, indução). O que me parece inicialmente mais relevante, para introduzir essa divisão em um contexto de investigação sobre artefatos, é a idéia de que sistemas semióticos estão imersos em ambientes de short-cuts construídos e/ou selecionados para obter informação sobre ícones, índices, símbolos, e combinações destas modalidades. Por exemplo, com respeito aos diagramas, que constituem uma classe de artefatos predominantemente icônico, eles são a principal, senão a única, forma de adquirirmos nova informação sobre relações uma vez que representam seus objetos através das relações entre as partes que os constituem. Parece um truísmo afirmar que a maneira mais eficaz de representar relações espaciais é por meio de diagramas, como mapas e frames. São artefatos que provêem um nicho construido para extração e manipulação de relações.

Minha argumentação é que o Design da Informação pode ser associado à criação, desenvolvimento e análise de artefatos semióticos, seus usuários a sistemas semióticos, os ambientes resultantes a nichos e paisagens semióticas. Embora pareça intuitivamente tratável a noção de que estamos imersos em um espaço mais ou menos estruturado de signos como artefatos, as implicações e consequências decorrentes deste conjunto de premissas encontra-se ainda por ser explorado. O resultado desta exploração pode prover uma agenda poderosa de pesquisa e investigação em Design da Informação.

\section{Referências}

BERGMAN, M. 2000. Reflections on the role of the communicative sign in semeiotic. Transactions of the Charles S. Peirce Society: A Quarterly Journal in American Philosophy, v. 36, n. 2: 225-254.

CLARK, A. 2003. Natural born-cyborg. Oxford: Oxford University Press.

2006. Language, embodiment, and the cognitive niche. In: Trends in Cognitive Sciences, v. 10 , n. 8, p.370-374.

_ 2008. Supersizing the Mind. Oxford University Press.

DASCAL, M. 2002. Language as a cognitive technology. International Journal of Cognition and Technology, v. 1, n. 1: 35-89.

DE TIENNE, A. 2003. Learning qua semiosis. S.E.E.D. Journal -- Semiotics, Evolution, Energy, and Development, n. 3: 37-53.

EMMECHE, C. 2006. A-life, organism and body: the semiotics of emergent levels. In: Farias, P. \& Queiroz, J. (Eds.) Advanced Issues on Cognitive Science and Semiotics: 5-18. Shaker Verlag.

FETZER, J. H. 1997. Thinking and computing: computers as special kinds of signs. Minds and Machines, n. 7: 345-364.

GRAU, O. 2003. Virtual Art: From Illusion to Immersion. Cambridge, Mass.: MIT Press/Leonardo Books.

HUTCHINS, E. 1999. Cognitive Artifacts. In: Wilson, R.A. \& Keil, F.C. (Eds.). The MIT Encyclopedia of the Cognitive Sciences: 126-7. Cambridge: MIT Press.

KIRSH, D. 2009. Problem Solving and Situated Cognition. In: Robbins, P \& Aydede, M. (Eds.) The Cambridge Handbook of Situated Cognition: 264-306. Cambridge: Cambridge University Press.

_ 2010. Thinking With External Representations. Al \& Society, v. 25, n. 4.

NORMAN, D. A. 1993. Things That Make Us Smart. Reading: Addison Wesley.

PEIRCE, Charles S. 1893-1913. The Essential Peirce. Selected Philosophical Writings. Vol. 2. THE PEIRCE EDITION PROJECT, (ed.) 1998. Bloomington \& Indianapolis: Indiana University Press. (citado como EP, seguido pelo número do volume e da página) 
1931-1935. The Collected Papers of Charles Sanders Peirce. [Electronic edition reproducing Vols. I-VI, HARTSHORNE, C. \& WEISS, P. (Eds.); Vols. VII-VIII, BURKS, A. W. (Ed.), Cambridge: Harvard University Press.] Charlottesville: Intelex Corporation. (citado como CP, seguido por volume e número do parágrafo)

1976. New elements of mathematics by Charles S. Peirce. C. Eisele (Ed.). Berlin: Mouton. (citado como NEM, seguido pelo número do volume e da página)

QUEIROZ, J. \& EL-HANI, C. 2006a. Semiosis as an emergent process. Transaction of C.S.Peirce Society, v. 42, n. 1: 78-116.

_. 2006b. Towards a multi-level approach to the emergence of meaning in living systems. Acta Biotheoretica, n. 54: 179-206.

QUEIROZ, J.; EMMECHE, C.; KULL, K. \& EL-HANI, C. (prelo). The biosemiotic approach in biology: theoretical bases and applied models. In: Terziz, G. \& Arp, R. (Eds.) The Ashgate Companion to Contemporary Philosophy of Biology. Ashgate Publishing Limited Press.

QUEIROZ, J. \& MERRELL, F. 2006. Semiosis and pragmatism: toward a dynamic concept of meaning. Sign System Studies, v.34, n.1: 37-66.

2009. On Peirce's pragmatic notion of semiosis - a contribution for the design of meaning machines. Minds \& Machines, n.19: 129-143.

RANSDELL, J. 1977. Some leading ideas of Peirce's semiotic. Semiotica, v.19, n.3/4:157-178.

SHORT, T. 2007. Peirce's Theory of Signs. Cambridge University Press.

SINHA, C. 2009. Language as a biocultural niche and social institution. In: Evans, V. \& Pourcel, S. (Eds.) New Directions in Cognitive Linguistics: 289-310. Amsterdam: John Benjamins.

STERELNY, K. 2008. Language and Niche Construction. In: GRIEBEL, U. \& OLLER, K. (Eds) From Fixed Signals to Contextually Free Communication: The Evolution of Complex Communication in Animals and Humans: 215-232. Cambridge, Mass.: MIT Press.

STJERNFELT, F. 2007. Diagrammatology - An Investigation on the Borderlines of Phenomenology, Ontology, and Semiotics. Springer Verlag.

TOMASELLO, M.; CARPENTER, M.; CALL, J.; BEHNE, T. \& MOLL, H. 2005. Understanding and sharing intentions: The origins of cultural cognition. In: Behavioral and Brain Sciences, $\mathrm{n}$. 28, p. 675 - 691.

VON UEXKÜLL, J. 1940. Bedeutungslehre. Bios 10. Leipzig: Johann Ambrosius Barth [= Thure von Uexküll (tradução),1982. The theory of meaning. Semiotica v. 42 n. 1: 25-82].

VON UEXKÜLL, T. 1982a. Introduction: Meaning and science in Jakob von Uexküll_s concept of biology. Semiotica, v. 42, n. 1: 1-24.

ZHANG, J. \& WANG, H. 2009. An exploration of the relations between external representations and working memory. PLoS ONE v.4, n.8: e6513.

\section{Sobre o autor}

João Queiroz, doutor em Comunicação e Semiótica pela PUC-SP, é professor no Instituto de Artes e Design e no Programa de Pós-Graduação em Comunicação (UFJF). Ele é editor do periódico International Journal of Signs and Semiotic Systems (http://www.igi-pub.com/ijsss). Seus principais interesses são: emergência e evolução de sistemas semióticos, artefatos e tecnologias cognitivas, semiótica e pragmatismo de Peirce.

<queirozj@pq.cnpq.br> 